\title{
Data-Driven Economy: Challenges and Opportunities
}

The transformation of data into an independent and strategic asset poses challenges for companies, regulators and policymakers alike. On 17 June 2019 in Berlin, together with the German Economic Institute (IW), Intereconomics hosted a conference on the "Data-Driven Economy" to reflect on the challenges and opportunities of competition policy, business models and data governance.

In order to guide the data-driven economy to benefit the greater good, regulators and policymakers will have to refine an often inefficient legal and regulatory framework. On the business side, tools such as marketplaces are needed to utilise data as an asset and monetise data while taking privacy and other considerations into account.

In their contributions to the conference and in this issue, participants discuss their perspectives on - and varying models of - data governance. From the role data governance plays as an enabler of the data economy to its role in enhancing innovation and welfare, our discussants clearly showed that there is no 'one-size-fits-all' model of this concept. Looking at data governance from a management as well as a societal perspective, participants defined its terminology and provided examples of its application. The conference addressed the growing need for a comprehensive view of data, a conversation that will undoubtedly continue to evolve.

\section{Competition and Competition Policy in a Data-Driven Economy}

Justus Haucap, Düsseldorf Institute for Competition Economics (DICE), Germany.

\section{Data Marketplaces: Trends and Monetisation of Data Goods}

Markus Spiekermann, Fraunhofer Institute for Software and Systems Engineering, Dortmund, Germany.

\section{Data Governance as the Enabler of the Data Economy}

Barbara Engels, German Economic Institute, Cologne, Germany.

\author{
Alternative Data Governance Models: Moving Beyond One-Size-Fits-All Solutions \\ Bruno Carballa Smichowski, Chronos, Paris; CEPN-Université Paris XIII, France.
}

\section{Data Governance: Enhancing Innovation and Protecting Against Its Risks}

Max von Grafenstein, Alexander von Humboldt Institute for Internet and Society, Berlin; Einstein Center Digital Future, Berlin, Germany.

Alina Wernick, Alexander von Humboldt Institute for Internet and Society, Berlin; Ludwig Maximilian University, Munich, Germany.

Christopher Olk, Alexander von Humboldt Institute for Internet and Society, Berlin; Technische Universität, Berlin, Germany. 